\title{
Ten-day high-dose proton pump inhibitor triple therapy versus sequential therapy for Helicobacter pylori eradication
}

\author{
Chonticha Auesomwang ${ }^{1}$, Monthira Maneerattanaporn ${ }^{1}$, William D. Chey ${ }^{3}$, Pattarachai Kiratisin ${ }^{2}$, Somchai \\ Leelakusolwong $^{1}$, Tawesak Tanwandee ${ }^{1}$
}

\begin{abstract}
${ }^{1}$ Division of Gastroenterology, Department of Medicine, Faculty of Medicine, Siriraj Hospital, Bangkok, Thailand ${ }^{2}$ Department of Microbiology, Faculty of Medicine, Siriraj Hospital, Bangkok, Thailand

${ }^{3}$ Division of Gastroenterology, Department of Medicine, University of Michigan, Ann Arbor, MI, USA
\end{abstract}

Corresponding author:

Monthira Maneerattanaporn, MD., MS. (CRDSA)

Division of Gastroenterology, Department of Internal Medicine, Siriraj Hospital, Mahidol University, Bangkok 10170, Thailand.

E-mail: monthira.man@mahidol.ac.th

Phone: +662-419-7261

Fax: +662-411-5013

\section{Declaration of personal interests: None}

Declaration of funding interests: The study medications including lansoprazole were provided by Takeda (Thailand) Ltd. The company was not involved in the study design, patient recruitment, assessment, data management, or manuscript preparation. Supporting funds were received from a Siriraj Chalermprakiat Grant, Mahidol University, and the Gastroenterological Association of Thailand.

Acknowledgments: The authors express sincere thanks to all volunteers and patients in this study. We also thank Susan Furness, PhD, from Edanz Group (www.edanzediting.com/ac) for editing a draft of this manuscript.

This is the author manuscript accepted for publication and has undergone full peer review but has not been through the copyediting, typesetting, pagination and proofreading process, which may lead to differences between this version and the Version of Record. Please cite this article as doi: $10.1111 /$ jgh.14292

This article is protected by copyright. All rights reserved. 


\begin{abstract}
Background/Aim

Eradication rates of Helicobacter pylori following standard triple therapy are declining worldwide, but high-dose proton-pump inhibitor-based triple therapy (HD-PPI-TT) and sequential therapy (ST) have demonstrated higher cure rates. We aimed to compare the efficacy and tolerability of HD-PPI-TT and ST in H. pylori-associated functional dyspepsia (HP-FD).
\end{abstract}

\title{
Methods
}

One hundred and twenty HP-FD patients were randomized to receive 10-day HD-PPI-TT (60 mg lansoprazole/500 mg clarithromycin/1 $\mathrm{g}$ amoxicillin, each administered twice daily for 10 days) or 10-day ST (30 mg lansoprazole/1 $\mathrm{g}$ amoxicillin, each administered twice daily for 5 days followed by $30 \mathrm{mg}$ lansoprazole/500 mg clarithromycin/400 mg metronidazole, each administered twice daily for 5 days). H. pylori status was determined in post-treatment week 4 by ${ }^{14} \mathrm{C}$-urea breath test. Eradication and antibiotic resistance rates, dyspeptic symptoms, drug compliance, and adverse effects were compared.

\section{Results}

Intention-to-treat (ITT) eradication rates were similar in the ST and HD-PPI-TT groups (85\% vs. $80 \% ; P=0.47)$. However, the eradication rate was significantly higher following ST compared with HD-PPI-TT in per protocol (PP) analysis ( $94.4 \%$ vs. $81.4 \%$; $P=0.035)$. ST achieved higher cure rates than HD-PPI-TT in clarithromycin-resistant $H$. pylori strains (100\% vs. $33.3 \%$; $P=0.02)$. Treatment compliance was similar in the HD-PPI-TT and ST groups, although nausea and dizziness were more common in the ST group.

\section{Conclusions}

ST achieved better $H$. pylori eradication than HD-PPI-TT in patients with FD. However, the eradication rate for ST fell from $94.4 \%$ in PP to $85 \%$ in ITT analysis. Adverse effects might result in poorer compliance and compromise actual ST efficacy. (ClinicalTrials.gov: NCT01888237). 
Key words: dyspepsia, proton pump inhibitors, Helicobacter pylori

\section{INTRODUCTION}

Helicobacter pylori plays an important role in the pathogenesis of chronic gastritis, peptic ulcer, and gastric cancer, and its successful eradication alters the natural history of these conditions. ${ }^{1,2}$ Both the Thai and Second Asia-Pacific Consensus Guidelines for H. pylori 2009 currently recommend proton-pump inhibitor-(PPI) based triple therapy (TT), referred to as standard TT (standard dose PPI + clarithromycin + amoxicillin or metronidazole), as frontline treatment for H. pylori eradication. ${ }^{3,4}$ However, the efficacy of this regimen has reportedly decreased to 50\%$75 \%$ worldwide, with clarithromycin-resistance recognized as a major cause of this treatment failure. ${ }^{5,6}$

Sequential therapy (ST), consisting of standard-dose PPI + amoxicillin for the first 5 days followed by 5 additional days of PPI + clarithromycin + metronidazole, reportedly increases the efficacy of $H$. pylori eradication and is now recommended as the first-line regimen in some guidelines. ${ }^{3,4,7,8}$ A recent meta-analysis revealed that $\mathrm{ST}$ achieved a higher eradication rate than standard TT, ${ }^{9}$ with eradication rates of up to $95 \%$ reported for ST in Thailand. ${ }^{10}$ However, ST is a complicated regimen compared with TT, with implications for patient compliance and facilitating the development of drug resistance. ${ }^{11}$

Evidence suggests that a sustained higher intragastric $\mathrm{pH}$ is a major therapeutic determinant of H. pylori eradication. ${ }^{12}$ Other factors, including inflammatory cytokine polymorphisms, especially the interleukin-1B-511 T/T genotype which is responsible for more rapid PPI metabolism, can also negatively affect eradication rates. ${ }^{13}$ Higher doses of PPIs have thus been advocated in the hope of maximizing $H$. pylori eradication. A recent meta-analysis showed that high-dose PPI triple therapy (HD-PPI-TT) eradicated $H$. pylori more effectively than TT with standard doses of PPI. ${ }^{14}$ Moreover, Vilaichone et al. reported that 14-day HD-PPI-TT achieved an excellent cure rate of up to $100 \%$, regardless of the CYP2C19 genotype. ${ }^{15}$ These findings, together with the fact that the regimen is simpler and more familiar to physicians than ST, make HD-PPI-TT an attractive therapeutic option. 
This study aimed to compare the efficacies of ST and HD-PPI-TT in patients with H. pylori associated functional dyspepsia. We hypothesized that both regimens would show comparable efficacies for eradicating $H$. pylori. Secondary outcomes included changes in dyspeptic symptom scores at 4, 8, and 24 weeks, compliance rates, adverse events, and the prevalence of clarithromycin-resistant $H$. pylori.

\section{METHODS}

\section{Design overview}

This was a prospective, randomized, controlled, open-label study including consecutive patients who met the inclusion and exclusion criteria. All participants provided written informed consent and were randomly assigned to one of two treatment groups. Follow-up visits were scheduled 4 weeks after completing treatment to determine eradication rates, treatment compliance, and the incidence of adverse events. The study was approved by the ethics committees of the participating center. (ClinicalTrials.gov: NCT01888237).

\section{Patients}

Consecutive patients attending a tertiary care hospital in Bangkok, Thailand, between May 2013 and March 2014 with functional dyspepsia, including epigastric pain and post-prandial distress syndrome, defined by the Rome III criteria, were enrolled. All patients who presented with at least one of four cardinal dyspepsia symptoms (postprandial fullness, early satiation, epigastric pain, and epigastric burning) were recruited. ${ }^{16}$ Eligible patients had compatible symptoms and had undergone esophagogastroduodenoscopy (EGD) with no evidence of peptic ulcer disease, gastric cancer, or erosive esophagitis. All patients had active $H$. pylori infection documented by a positive rapid urease test. Patients were at least 18 years old and had not previously been treated for $\mathrm{H}$. pylori infection. PPIs or histamine $\mathrm{H}_{2}$ receptor antagonists were withheld for at least 2 weeks prior to EGD. Exclusion criteria were: (i) use of non-steroidal anti-inflammatory drugs or antibiotics within the 2 weeks prior to EGD; (ii) current use of anticoagulants or ketoconazole; (iii) contraindication(s) to gastric biopsy; (iv) history of gastric surgery; (v) severe or unstable cardiovascular, pulmonary, or endocrine disease; clinically significant renal or hepatic disease or dysfunction; hematological disorders; AIDS; stroke (bed ridden); (vi) pregnancy or lactation; and (vii) allergy to any of the study drugs. 


\section{Randomization and interventions}

Patient allocation was performed using a computer-generated randomization chart. Patients were randomly allocated to receive a 10-day course of HD-PPI-TT (60 mg lansoprazole, $500 \mathrm{mg}$ clarithromycin, and $1 \mathrm{~g}$ amoxicillin, each administered twice daily for 10 days) or a 10-day course of ST (30 mg lansoprazole, $1 \mathrm{~g}$ amoxicillin, each administered twice daily for the first 5 days, followed by $30 \mathrm{mg}$ lansoprazole, $500 \mathrm{mg}$ clarithromycin, and $400 \mathrm{mg}$ metronidazole, each administered twice daily for the remaining 5 days). The required medications were supplied in individual blister packs with detailed instructions on how to take them.

\section{Measurements and outcomes}

The primary outcome of the study was comparative $H$. pylori eradication rates in patients receiving ST and HD-PPI-TT regimens. Secondary outcomes were adherence and adverse events in the two study groups. The impact of clarithromycin resistance on eradication rates of ST and HD-PPI-TT was also assessed. ${ }^{17}$ Notably, we did not collect data on metronidazole or amoxicillin resistance. Dyspeptic symptoms were assessed in all participants before and 6 months after treatment of $H$. pylori by face to face interviews in our hospital clinic, or by phone interview in patients unable to attend a meeting. A modified SF-Leeds dyspepsia score was used to evaluate upper gastrointestinal symptoms at baseline, and at weeks 4, 8, and 24 after completion of treatment. ${ }^{18}$ Symptoms assessed included epigastric pain/discomfort, heartburn, acid regurgitation, and nausea and vomiting. Symptom severity was scored from 0 (no symptoms) to 4 (more than once a day).

\section{${ }^{14}$ C-Urea breath test}

Urease activity was detected as a surrogate marker of $H$. pylori infection by ${ }^{14} \mathrm{C}$-urea breath test using a PYtest kit (Kimberley-Clark, Roswell, GA, USA) and a Microcount scintillation counter (Halyard Health, Alpharetta, GA, USA). After at least $6 \mathrm{~h}$ fasting, participants were asked to ingest a PYtest ${ }^{14} \mathrm{C}$-labeled urea capsule with $30 \mathrm{~mL}$ of water, followed by an additional $20 \mathrm{~mL}$ of water $3 \mathrm{~min}$ later. Single-breath samples were then collected $7 \mathrm{~min}$ later by exhaling into Mylar breath-collection balloons after holding their breath for $5-10 \mathrm{~s}$. The amount of ${ }^{14} \mathrm{C}$ in the balloon was measured using a scintillation counter and reported as disintegrations per minute (DPM). Participants were classified as negative (uninfected) if their DPM value was $<50$. 


\section{Endoscopy and $H$. pylori detection}

All eligible participants underwent EGD during which gastric biopsies were collected from the antrum and incisura, respectively, and used for the rapid urease test (Kimberly-Clark). An additional five biopsy specimens were collected from each patient, including two from the antrum, two from the corpus, and one from the incisura, placed into sterile bottles and sent to the Department of Microbiology, Faculty of Medicine, Siriraj Hospital for culture. Patients with a positive rapid urease test were invited to participate in the study.

For bacterial culture and susceptibility testing, biopsy specimens were delivered directly to a single microbiological laboratory within $2 \mathrm{~h}$ of biopsy and then stored at $-70^{\circ} \mathrm{C}$. Isolated strains were obtained using Columbia or sheep blood agar in a microaerophilic environment and tested for primary clarithromycin resistance using the Epsilometer test (BioMérieux, Durham, NC, USA). The results were considered positive if the minimal inhibitory concentration was $>1$ $\mathrm{mg} / \mathrm{L}$.

\section{Follow-up procedures}

\section{Compliance with treatment and adverse events}

Patients were scheduled for follow-up visits to assess symptoms, compliance with therapy, and adverse events. Adverse events were evaluated by asking open-ended questions, followed by more specific questions relating to anticipated adverse effects. Compliance was pre-defined as the consumption of $>90 \%$ of the prescribed drugs and was determined by pill counts.

\section{Confirmation of eradication}

${ }^{14} \mathrm{C}$-urea breath tests were performed at least 4 weeks after completion of treatment and infection was considered to have been eradicated when the result was $<50$ DPM.

\section{Statistical analysis}

We calculated the sample size needed to detect a difference in eradication rates between ST (assumed to have an eradication rate of 97\%) and HD-PPI-TT (assumed to have an eradication rate of $82 \%$ ) of $15 \%$, with power of $80 \%$ and significance level of 0.05 (alpha $=0.05$, two- 
sided). ${ }^{8}{ }^{14}$ A $10 \%$ dropout rate was anticipated. The minimum required sample size was calculated to be 59 patients per group.

The primary study outcome was a comparison of $H$. pylori eradication rates between the ST and HD-PPI-TT regimens using the intention to treat (ITT) population. A secondary per protocol (PP) analysis was also performed. Categorical variables were reported as numbers and percentages. Eradication rates were compared between the groups using $\chi^{2}$ and Fisher's exact tests. The $95 \%$ confidence intervals for the eradication rate ratio and difference in absolute risk between the two groups were calculated. The secondary outcome was the impact of clarithromycin resistance on eradication rates, and adherence and adverse events were reported as numbers and percentages and compared between the ST and HD-PPI-TT groups using $\chi^{2}$ and Fisher's exact tests. The composite dyspepsia scores at each time point from baseline to 24 weeks after treatment were compared using Student's $t$-tests. All statistical analyses were performed using SPSS version 18.0 (SPSS Inc., Chicago, IL, USA). A $P$ value $<0.05$ was considered statistically significant.

\section{RESULTS}

\section{Study population}

The patient flow chart is shown in Figure 1. The mean patient age ( \pm standard deviation) was 49.6 \pm 11.1 years. The baseline characteristics of the patients were similar in both groups (Table 1). Among the 120 randomly assigned patients, seven were excluded from the PP analysis, including one in the HD-PPI-TT group because of adverse events and six in the ST group because of loss to follow-up ( $n=2)$ and adverse events $(n=4)$. Sixty patients in the HD-PPI-TT and 60 in the ST group were finally included in the ITT analysis, and 59 patients in the HD-PPITT and 54 in the ST group were available for PP analysis. More patients were excluded from the ST than the HD-PPI-TT group, but the difference was not significant $(6 / 60=10 \%$ and $1 / 60=$ $1.67 \%$, respectively; $P=0.11)$.

\section{Eradication of $\mathrm{H}$. pylori infection}

The overall eradication rates of the two treatments according to ITT and PP are summarized in Table 2 . The eradication rates were $80 \%$ in the HD-PPI-TT group and $85 \%$ in the ST group $(P=$ 
0.47) according to primary ITT analysis, and $81.4 \%$ and $94.4 \%$, respectively, according to PP analysis $(P=0.035)$.

\section{Role of clarithromycin resistance in eradication of $\boldsymbol{H}$. pylori infection}

Ninety of the 120 study subjects $(75 \%)$ had positive $H$. pylori cultures, with similar proportions in both treatment groups (48/60 [80\%] in the HD-PPI-TT and 42/60 [70\%] in the ST group; $P=$ 0.21). Nine of the 90 patients (10\%) had clarithromycin-resistant strains of $H$. pylori. The eradication rates of the two treatments stratified by clarithromycin resistance are shown in Table 3. Clarithromycin-resistant $H$. pylori was eradicated in one of three $(33.3 \%)$ patients treated with HD-PPI-TT versus six out of six $(100 \%)$ treated with ST $(P=0.02)$. The eradication rates in patients with clarithromycin-susceptible $H$. pylori strains were similar for both treatments (86.7\% vs. $83.3 \%$ for HD-PPI-TT and ST, respectively; $P=0.68)$.

\section{Treatment compliance}

A total of 59/60 patients (98.3\%) in the HD-PPI-TT and 54/58 (93.1\%) in the ST group complied with the allocated treatment ( $>90 \%$ of medication taken according to pill count). There was no significant difference in compliance between the groups $(P=0.20)$.

\section{Adverse events}

There was no significant difference in the incidence of adverse events reported by the two treatment groups, $(P=0.20)$ (Table 4). However, there was a tendency towards a higher rate of adverse events in the ST group, with taste disturbance, dry mouth, and nausea being the most commonly reported events. The most common adverse events in the HD-PPI-TT group were taste disturbance, dry mouth, and abdominal bloating. Four patients in the ST and one in the HDPPI-TT group discontinued treatment because of adverse events including taste disturbance, dizziness, nausea, and vomiting. All patients in the ST group who discontinued their medication did so during the last 5 days, when they were taking metronidazole and clarithromycin.

\section{Dyspeptic symptoms}

The median composite dyspepsia scores decreased gradually over time in both groups $(P<$ 0.001), with no significant differences in scores between the two groups (ITT analysis: Figure 
2A, PP analysis: Figure 2B). Furthermore, this pattern was similar in patients with and without successful $H$. pylori eradication (Figure 3).

\section{DISCUSSION}

Many guidelines currently recommend standard TT as the first-line treatment for eradication of H. pylori infection. ${ }^{4,8,19}$ Although early studies of first-line standard TT demonstrated eradication rates of $>85 \%,{ }^{20}$ a meta-analysis suggested that the current eradication rate is likely to be $<80 \%,{ }^{21}$ with increased antibiotic resistance, particularly to clarithromycin, being responsible for this decline. ${ }^{5}$ Reported rates of clarithromycin resistance in Thailand have ranged from $3.5 \%-23 \%$ over the past 13 years. The current study, conducted in a tertiary care facility, identified a $10 \%$ prevalence of clarithromycin resistance amongst $H$. pylori strains.

ST has proven superior to 7-10 days of standard TT for eradicating H. pylori. ${ }^{8,22-25}$ It has been suggested that $H$. pylori can develop efflux channels to remove clarithromycin rapidly from the bacterial cell. ${ }^{26}$ Given that amoxicillin acts on and weakens the bacterial cell wall, it is possible that this initial phase of treatment may prevent the development of efflux channels, thus improving the efficacy of clarithromycin in the second phase of treatment. ${ }^{26} \mathrm{It}$ is also possible that the efficacy of ST may be related to the use of three, rather than two antibiotics. ${ }^{27}$ However, the increased complexity of ST and consequently negative effects on compliance are potential concerns of this type of regimen. ${ }^{11}$

A meta-analysis of large, multicenter, randomized trials found that HD-PPI-TT was more efficacious than standard TT. ${ }^{14}$ Sugimoto et al. reported that cure rates were closely related to the degree of acid inhibition, with a mean gastric $\mathrm{pH}$ of 6.4 in patients with $H$. pylori eradication compared with 5.2 in those in those without eradication. ${ }^{12}$ Moreover, differences in CYP2C19 genotypes and the associated effects on PPI metabolism may affect the potency of acid inhibition ${ }^{28,29}$ However, methods for determining CYP2C19 genotype are not widely available, and the use of HD-PPI-TT offers a practical way of circumventing this issue.

Studies directly comparing HD-PPI-TT and ST are lacking, and the current study was the first prospective, randomized, controlled, open-label study to compare these two regimens in patients with $H$. pylori -positive functional dyspepsia. The eradication rates of the two regimens were similar according to primary ITT analysis (80\% HD-PPI-TT, $85 \%$ ST; $P=0.47$ ), but ST achieved 
a significantly higher eradication rate than HD-PPI-TT according to PP analysis (94.4\% vs. $81.4 \%, P=0.035$ ) (Table 2). The eradication rates in our study were similar to those reported in recent meta-analyses (97\% and $82 \%$ for ST and HD-PPI-TT, respectively). ${ }^{14,30}$ However, the eradication rate of $80 \%$ for HD-PPI-TT differed from that reported by Vilaichone et al., who found an eradication rate of $100 \%$ with this regimen. ${ }^{15}$ There are several possible explanations for these divergent eradication rates: firstly, our population had higher clarithromycin resistance (10\% compared with $0 \%$ ), and secondly, the duration of HD-PPI-TT was different (10 days in our study and 14 days in Vilaichone et al.).

Among the small subgroup of patients with clarithromycin-resistant $H$. pylori, the eradication rate was significantly lower in the HD-PPI-TT compared with the ST group (33\% vs. 100\%, respectively; $P=0.02$ ). These results are similar to those of other studies that reported greater efficacy of ST compared with TT in patients with clarithromycin-resistant H. pylori. ${ }^{8,31}$ However, given the small number of patients with clarithromycin-resistant $H$. pylori in this study we believe that it is premature to conclude that ST was superior to HD-PPI-TT based on the current data. Based on these observations, ST has been suggested as the optimal first-line therapy in regions with a prevalence of clarithromycin resistance $>15 \% .^{32}$

The current results regarding antimicrobial resistance should be interpreted with caution. The inability to culture $H$. pylori meant that complete antibiotic sensitivity tests were only carried out in $25 \%$ of patients, and the fact that only clarithromycin resistance was tested for in all patients thus limits the conclusions that can be drawn from this analysis. In particular, the failure to assess metronidazole resistance may be particularly important, given that metronidazole resistance has previously been shown to adversely affect the efficacy of sequential therapy. ${ }^{33}$ Nevertheless, the relatively high rate of eradication by ST (85\%) suggests that either there was a low rate of metronidazole resistance in the study population, or that metronidazole resistance had little effect on the efficacy of ST.

The current study included 11 patients in the HD-PPI-TT group and three in the ST group who did not achieve successful eradication of $H$. pylori infection. Although two patients with treatment failure in the HD-PPI-TT group had clarithromycin-resistant $H$. pylori, treatment also failed in nine patients in the HD-PPI-TT and three in the ST group, despite the absence of clarithromycin resistance. This might be explained by amoxicillin or metronidazole resistance, 
which was not assessed as part of this study. Our results thus suggest that the ability of ST to eradicate $H$. pylori was not substantively affected by clarithromycin resistance. The reason for this observation is unclear, but it is consistent with previous reports from other parts of the world. $^{34,35}$

Poor compliance is the second most important reason after clarithromycin resistance for a failure to eradicate $H$. pylori. ${ }^{36}$ In a pooled-data analysis, Zullo et al. found no difference in compliance between ST and standard $\mathrm{TT}^{37}$ while another meta-analysis found no difference in compliance between HD-PPI-TT and standard TT. ${ }^{14}$ In our study, $98.3 \%$ of patients in the HD-PPI-TT and 93.1\% in the ST group complied with the treatment, which was consistent with these previous studies.

Notably, this was the first study to compare HD-PPI-TT and ST directly. Although the overall incidence of adverse events did not differ significantly between the two regimens $(P=0.06)$, there was a trend towards a higher incidence of adverse events and discontinuations in the ST group.

Whether or not $H$. pylori eradication improves functional dyspepsia remains controversial, though recent meta-analyses suggested a small but statistically significant benefit. ${ }^{38-40}$ The current study found that participants experienced a reduction in dyspeptic symptoms, measured by composite dyspepsia scores, at each time point between baseline and 24 weeks, regardless of whether their $H$. pylori was eradicated or not. However, the very small number of patients with persistent $H$. pylori infection following eradication therapy meant that it was not possible to make meaningful between-group comparisons.

This study had several limitations. Firstly, the study was not blinded to patients or providers, creating the possibility of bias. Secondly, very few patients had clarithromycin-resistant $H$. pylori, and the results of this secondary analysis should thus be interpreted with caution. We did not test for amoxicillin or metronidazole resistance, and we were therefore unable to assess the potential impacts of these on the efficacies of the treatments. Furthermore, we did not analyze CYP2C19 genotypes, which could have been particularly relevant to the results for the HD-PPITT group. Finally, it would have been interesting to include a treatment arm with a potassium- 
competitive acid blocker, which has recently been approved as part of a triple therapy regimen for first-line HP eradication in Japan. ${ }^{41}$

In conclusion, we report the first randomized trial comparing the efficacy of HD-PPI-TT and ST in patients with HP-positive functional dyspepsia. Although the regimens showed similar efficacy according to ITT analysis, ST achieved higher eradication rates than HD-PPI-TT amongst patients who completed the prescribed treatment. ST may also offer incremental benefits in patients infected with clarithromycin-resistant HP. (ClinicalTrials.gov Identifier: NCT01888237)

\section{REFERENCES}

1. Suerbaum S, Michetti P. Helicobacter pylori infection. N Engl J Med 2002; 347: 11771186.

2. Vakil N, Megraud F. Eradication therapy for Helicobacter pylori. Gastroenterology 2007; 133: 985-1001.

3. Gastroenterology Association of Thailand. Guidelines for the Management of Dyspepsia and Helicobacter pylori. $1^{\text {st }}$ ed. 2010: 1-30.

4. Fock KM, Katelaris P, Sugano K, et al. Second Asia-Pacific Consensus Guidelines for Helicobacter pylori infection. J Gastroenterol Hepatol 2009; 24: 1587-1600.

5. Mahachai V, Thong-Ngam D, Noophun P, et al. Efficacy of clarithromycin-based triple therapy for treating Helicobacter pylori in Thai non-ulcer dyspeptic patients with clarithromycin-resistant strains. J Med Assoc Thai 2006; 89(Suppl. 3): 74-78.

6. Megraud F. H pylori antibiotic resistance: prevalence, importance, and advances in testing. Gut 2004; 53: 1374-1384.

7. Sanchez-Delgado J, Calvet X, Bujanda L, et al. Ten-day sequential treatment for Helicobacter pylori eradication in clinical practice. Am J Gastroenterol 2008; 103: 22202223.

This article is protected by copyright. All rights reserved. 
8. Chey WD, Wong BC. Practice Parameters Committee of the American College of Gastroenterology guideline on the management of Helicobacter pylori infection. Am J Gastroenterol 2007; 102: 1808-1825.

9. Gatta L, Vakil N, Leandro G, et al. Sequential therapy or triple therapy for Helicobacter pylori infection: systematic review and meta-analysis of randomized controlled trials in adults and children. Am J Gastroenterol 2009; 104: 3069-3079.

10. Sirimontaporn N, Thong-Ngam D, Tumwasorn S, et al. Ten-day sequential therapy of Helicobacter pylori infection in Thailand. Am J Gastroenterol 2010; 105: 1071-1075.

11. Yang YJ, Sheu BS. Sequential therapy in childhood Helicobacter pylori eradication: emphasis on drug compliance. J Pediatr 2011; 159: 700; author reply 700-701.

12. Sugimoto M, Furuta T, Shirai N, et al. Evidence that the degree and duration of acid suppression are related to Helicobacter pylori eradication by triple therapy. Helicobacter 2007; 12: 317-323.

13. Sugimoto M, Furuta T, Yamaoka Y. Influence of inflammatory cytokine polymorphisms on eradication rates of Helicobacter pylori. J Gastroenterol Hepatol 2009; 24: 1725-1732.

14. Villoria A, Garcia P, Calvet X, et al. Meta-analysis: high-dose proton pump inhibitors vs. standard dose in triple therapy for Helicobacter pylori eradication. Aliment Pharmacol Ther 2008; 28: 868-877.

15. Prasertpetmanee S, Mahachai V, Vilaichone RK. Improved efficacy of proton pump inhibitor-amoxicillin-clarithromycin triple therapy for Helicobacter pylori eradication in low clarithromycin resistance areas or for tailored therapy. Helicobacter 2013; 18: 270273.

16. Tack J, Talley NJ, Camilleri $\mathrm{M}$, et al. Functional gastroduodenal disorders. Gastroenterology 2006; 130: 1466-1479.

17. Tongtawee T, Dechsukhum C, Matrakool L. High prevalence of Helicobacter pylori resistance to clarithromycin: a hospital-based cross-sectional study in Nakhon Ratchasima Province, Northeast of Thailand. Asian Pac J Cancer Prev 2015; 16: 8281-8285.

This article is protected by copyright. All rights reserved. 
18. Fraser A, Delaney BC, Ford AC, et al. The Short-Form Leeds Dyspepsia Questionnaire validation study. Aliment Pharmacol Ther 2007; 25: 477-486.

19. Malfertheiner P, Megraud F, O’Morain C, et al. Current concepts in the management of Helicobacter pylori infection: the Maastricht III Consensus Report. Gut 2007; 56: 772781.

20. Malfertheiner P, Megraud F, O'Morain C, et al. Current concepts in the management of Helicobacter pylori infection--the Maastricht 2-2000 Consensus Report. Aliment Pharmacol Ther 2002; 16: 167-180.

21. Laheij RJ, Rossum LG, Jansen JB, et al. Evaluation of treatment regimens to cure Helicobacter pylori infection--a meta-analysis. Aliment Pharmacol Ther 1999; 13: 857864.

22. Zhou L, Zhang J, Chen M, et al. A comparative study of sequential therapy and standard triple therapy for Helicobacter pylori infection: a randomized multicenter trial. Am J Gastroenterol 2014; 109: 535-541.

23. Kate V, Kalayarasan R, Ananthakrishnan N. Sequential therapy versus standard triple-drug therapy for Helicobacter pylori eradication: a systematic review of recent evidence. Drugs 2013; 73: 815-824.

24. Ang TL, Fock KM, Song M, et al. Ten-day triple therapy versus sequential therapy versus concomitant therapy as first line treatment for Helicobacter pylori infection. J Gastroenterol Hepatol 2015; 30: 1134-1139.

25. Greenberg ER, Anderson GL, Morgan DR, et al. 14-day triple, 5-day concomitant, and 10day sequential therapies for Helicobacter pylori infection in seven Latin American sites: a randomised trial. Lancet 2011; 378: 507-514.

26. De Francesco V, Margiotta M, Zullo A, et al. Clarithromycin-resistant genotypes and eradication of Helicobacter pylori. Ann Intern Med 2006; 144: 94-100.

This article is protected by copyright. All rights reserved. 
27. Tsay FW, Wu DC, Kao SS, et al. Reverse sequential therapy achieves a similar eradication rate as standard sequential therapy for Helicobacter pylori eradication: a randomized controlled trial. Helicobacter 2015; 20: 71-77.

28. Furuta T, Shirai N, Takashima M, et al. Effect of genotypic differences in CYP2C19 on cure rates for Helicobacter pylori infection by triple therapy with a proton pump inhibitor, amoxicillin, and clarithromycin. Clin Pharmacol Ther 2001; 69: 158-168.

29. Sugimoto M, Furuta T. Efficacy of tailored Helicobacter pylori eradication therapy based on antibiotic susceptibility and CYP2C19 genotype. World J Gastroenterol 2014; 20: 6400-6411.

30. Scaccianoce G, Hassan C, Panarese A, et al. Helicobacter pylori eradication with either 7day or 10-day triple therapies, and with a 10-day sequential regimen. Can J Gastroenterol 2006; 20: 113-117.

31. Tong JL, Ran ZH, Shen J, et al. Sequential therapy vs. standard triple therapies for Helicobacter pylori infection: a meta-analysis. J Clin Pharm Ther 2009; 34: 41-53.

32. Gisbert JP, Calvet X, O'Connor A, et al. Sequential therapy for Helicobacter pylori eradication: a critical review. J Clin Gastroenterol 2010; 44: 313-325.

33. Wang Y, Zhao R, Wang B, et al. Sequential versus concomitant therapy for treatment of Helicobacter pylori infection: an updated systematic review and meta-analysis. Eur J Clin Pharmacol 2018; 74: 1-13.

34. Gatta L, Scarpignato C, Fiorini G, et al. Impact of primary antibiotic resistance on the effectiveness of sequential therapy for Helicobacter pylori infection: lessons from a 5-year study on a large number of strains: Aliment Pharmacol Ther 2018; Epub ahead of print.

35. Dolapcioglu C, Koc-Yesiltoprak A, Ahishali E, et al. Sequential therapy versus standard triple therapy in Helicobacter pylori eradication in a high clarithromycin resistance setting. Int J Clin Exp Med 2014; 7: 2324-2328.

36. Huang JQ, Hunt RH. Treatment after failure: the problem of "non-responders". Gut 1999; 45(Suppl. 1): I40-I44.

This article is protected by copyright. All rights reserved. 
37. Zullo A, De Francesco V, Hassan C, et al. The sequential therapy regimen for Helicobacter pylori eradication: a pooled-data analysis. Gut 2007; 56: 1353-1357.

38. Moayyedi P, Soo S, Deeks J, et al. WITHDRAWN: Eradication of Helicobacter pylori for non-ulcer dyspepsia. Cochrane Database Syst Rev 2011.

39. Moayyedi P1, Soo S, Deeks J, et al. Eradication of Helicobacter pylori for non-ulcer dyspepsia. Cochrane Database Syst Rev 2006.

40. Du LJ, Chen BR, Kim JJ, et al. Helicobacter pylori eradication therapy for functional dyspepsia: Systematic review and meta-analysis. World J Gastroenterol 2016; 22: 34863495.

41. Matsumoto H, Shiotani A, Katsumata R, et al. Helicobacter pylori Eradication with proton pump inhibitors or potassium-competitive acid blockers: The effect of clarithromycin resistance. Dig Dis Sci 2016; 61: 3215-3220.

This article is protected by copyright. All rights reserved. 


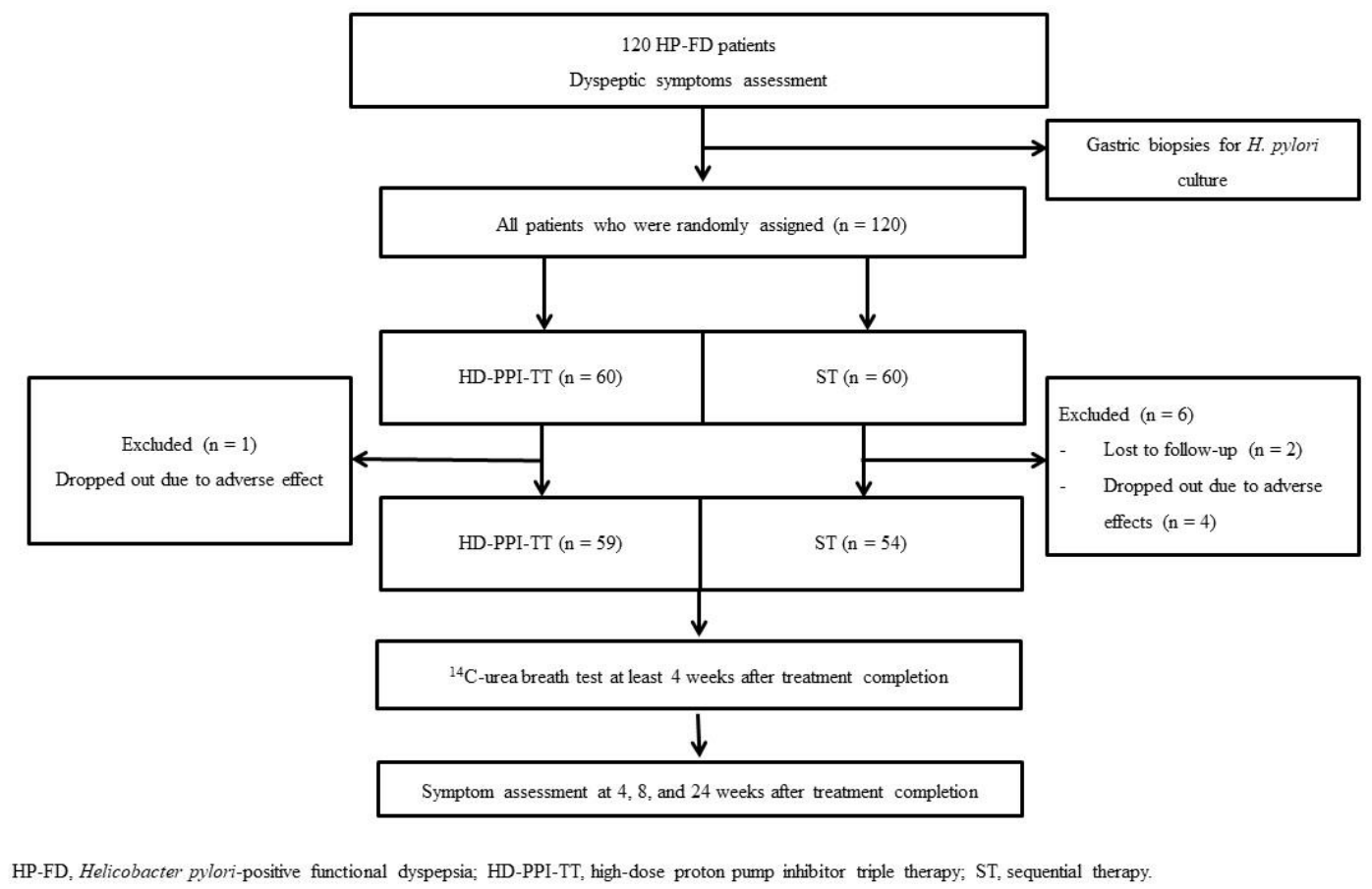

Figure 1 Study flow diagram

This article is protected by copyright. All rights reserved. 

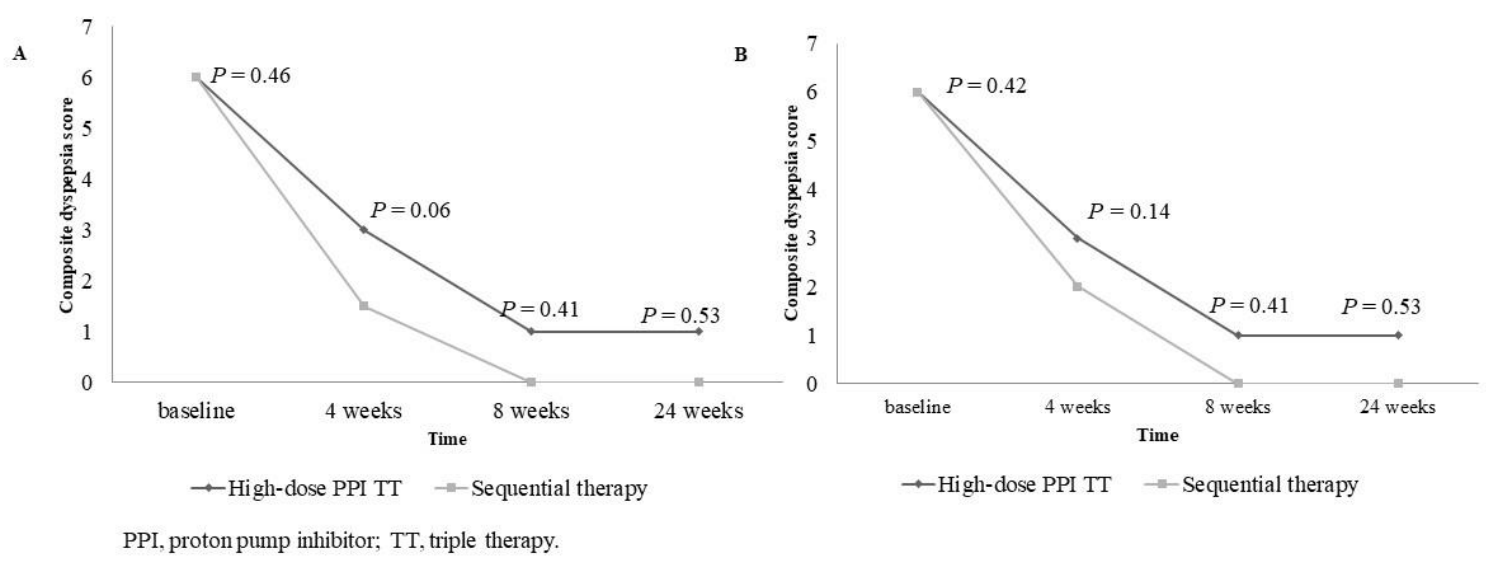

Figure 2 Composite dyspepsia scores at each time point from baseline to 24 weeks after treatment; ITT analysis (A) and PP analysis (B) 


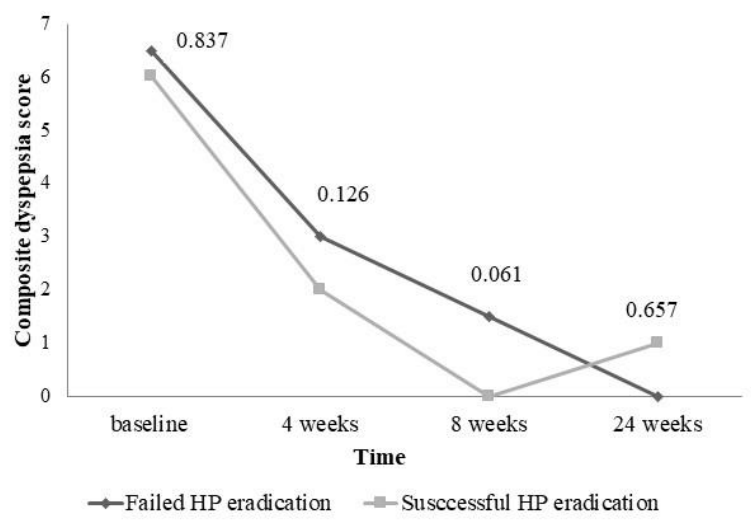

Figure 3 Composite dyspepsia scores at each time point from baseline to 24 weeks after treatment in patients with successful and failed Helicobacter pylori (HP) eradication 
Table 1 Baseline characteristics of the study population

\begin{tabular}{|l|c|c|c|}
\hline Variable & HD-PPI-TT (n= 60) & ST (n= 60) & P value \\
\hline Mean age (SD), years & $54.3(11.9)$ & $55.4(14.1)$ & 0.63 \\
\hline Patients, n (\%) & $28(46.7)$ & $18(30.0)$ & 0.06 \\
• Men & $32(53.3)$ & $42(70)$ & \\
\hline$\quad$ Women & 24.0 & 24.9 & 0.24 \\
\hline Mean body mass index, kg/m ${ }^{2}$ & $14(23.3)$ & $7(11.7)$ & 0.10 \\
\hline Patients who smoke, n (\%) & $23(38.3)$ & $14(23.3)$ & 0.08 \\
\hline Patients who drink alcohol, n (\%) & $48(80)$ & $42(70)$ & 0.21 \\
\hline H. pylori-positive culture, n (\%) & & & \\
\hline
\end{tabular}

HD-PPI-TT, high-dose proton pump inhibitor-based triple therapy; ST, sequential therapy. 
Table 2 Helicobacter pylori eradication rates in high-dose proton pump inhibitor based triple therapy (HD-PPI-TT) and sequential therapy (ST) groups.

\begin{tabular}{|c|c|c|c|c|c|c|}
\hline & \multicolumn{2}{|c|}{ HD-PPI-TT } & \multicolumn{2}{|c|}{ ST } & \multirow{2}{*}{$\begin{array}{c}\text { Difference } \\
(95 \% \mathrm{CI}) \text {, } \\
\%\end{array}$} & \multirow[t]{2}{*}{$\begin{array}{c}P \\
\text { value }\end{array}$} \\
\hline & Patients, $n$ & $\begin{array}{l}\text { Eradication } \\
\text { rate, n (\%) }\end{array}$ & Patients, $n$ & $\begin{array}{l}\text { Eradication } \\
\text { rate, n (\%) }\end{array}$ & & \\
\hline ITT & 60 & $48(80)$ & 60 & $51(85)$ & 1.7 & 0.47 \\
\hline $\mathbf{P P}$ & 59 & $48(81.4)$ & 54 & $51(94.4)$ & 11.1 & 0.035 \\
\hline
\end{tabular}

ITT, intention-to-treat; PP, per-protocol analysis

This article is protected by copyright. All rights reserved. 
Table 3 Effect of clarithromycin susceptibility on Helicobacter pylori eradication rates of both regimens (based on per-protocol analysis)

\begin{tabular}{cccc}
\hline Clarithromycin & HD-PPI-TT, n/n (\%) & ST, n/n (\%) & P value \\
\hline Resistant & $1 / 3(33.3)$ & $6 / 6(100)$ & $\mathbf{0 . 0 2}$ \\
Susceptible & $39 / 45(86.7)$ & $30 / 36(83.3)$ & 0.68 \\
\hline
\end{tabular}

HD-PPI-TT, high-dose proton pump inhibitor-based triple therapy; ST, sequential therapy

This article is protected by copyright. All rights reserved. 
Table 4 Adverse events for both treatment regimens

\begin{tabular}{|c|c|c|c|}
\hline Adverse event & HD-PPI-TT $(n=60), n(\%)$ & ST $(n=58), n(\%)$ & $P$ value \\
\hline Abdominal bloating & $9(15)$ & $6(10.3)$ & 0.45 \\
\hline Dizziness & $3(5)$ & $10(17.2)$ & 0.03 \\
\hline Dry mouth & $16(26.7)$ & $22(37.9)$ & 0.19 \\
\hline Fatigue & $5(8.3)$ & $8(13.8)$ & 0.34 \\
\hline Headache & $4(6.7)$ & $8(13.8)$ & 0.20 \\
\hline Nausea & $4(6.7)$ & $12(20.7)$ & 0.03 \\
\hline Taste disturbance & $33(55)$ & $37(63.8)$ & 0.33 \\
\hline Total & $38(63.3)$ & $46(79.3)$ & 0.06 \\
\hline $\begin{array}{c}\text { Drug withdrawal due to } \\
\text { adverse events }\end{array}$ & $1(1.7)$ & $4(6.9)$ & 0.20 \\
\hline
\end{tabular}

*Only adverse effects with an incidence $>5 \%$ were included in the table.

HD-PPI-TT, high-dose proton pump inhibitor-based triple therapy; ST, sequential therapy.

This article is protected by copyright. All rights reserved. 